in vivo $34: 2897-2903(2020)$

doi:10.21873/invivo.12118

\title{
Improvement in Left Cardiac Function Following Mitral Valve Repair: Analyses Based on Cardiac Magnetic Resonance Imaging
}

\author{
YOSHIHIRO HAYATA ${ }^{1}$, NORIYOSHI SAWABATA ${ }^{1}$, TAKEHISA ABE ${ }^{1}$, TOMOAKI HIROSE $^{1}$, \\ KEIGO YAMASHITA ${ }^{1}$, SHUN HIRAGA ${ }^{1}$, RYOHEI FUKUBA ${ }^{1}$, JUNICHI TAKEMURA ${ }^{1}$, \\ TOMOYA NAKANO ${ }^{2}$, YOSHIHIKO SAITO ${ }^{2}$ and SHIGEKI TANIGUCHI ${ }^{1}$ \\ ${ }^{1}$ Department of Thoracic and Cardiovascular Surgery, \\ Nara Medical University School of Medicine, Kashihara, Japan; \\ ${ }^{2}$ Department of Cardiovascular Medicine, Nara Medical University School of Medicine, Kashihara, Japan
}

\begin{abstract}
Aim: This study aimed to evaluate the structural and functional changes of left-sided cardiac chambers by cardiac magnetic resonance imaging (CMRI) in patients with chronic mitral regurgitation after mitral valve repair (MVR). Patients and Methods: Among 103 patients who underwent $M V R, 21$ showed normal left ventricular (LV) function; their pre-and postoperative left atrial (LA) and LV functions were examined by CMRI. Results: $L V$ end-diastolic volume, $L V$ end-systolic volume, and LV mass significantly were reduced postoperatively $(p<0.01)$ and postoperative $L V$ ejection fraction tended to decrease. LA volume parameters also significantly decreased postoperatively $(p<0.01)$. The conduit function positively affected the LV filling volume postoperatively $(p<0.01)$; however, no effect on the booster pump function was noted ( $p=0.01)$. Conclusion: Restoration of LA and LV functions after a successful MVR was not associated with structural improvement in LA and LV.
\end{abstract}

Mitral valve repair (MVR) is the gold standard technique for chronic mitral regurgitation (MR) and studies have reported its excellent long-term outcomes $(1,2)$. Currently, the indication of MVR is based on several findings, such as clinical symptoms, poor left ventricle (LV) systolic function,

This article is freely accessible online.

Correspondence to: Noriyoshi Sawabata, MD, Department of Thoracic and Cardiovascular Surgery, Nara Medical University School of Medicine, 840 Shijo-cho, Kashihara City, Nara 634-8521, Japan, Tel: +81 744223051 (Ext. 3423), Fax: +81 7448040, e-mail: nsawabata@naramed-u.ac.jp

Key Words: Mitral regurgitation, mitral valve repair, left ventricle, left atrium, cardiac magnetic resonance. atrial fibrillation, and higher pulmonary artery pressure, according to the American (3) and European practice guidelines (4). Evidence showed that symptom severity and hemodynamics are associated with patient outcomes (5). However, postoperative changes in left-sided cardiac chambers [i.e., the left atrium (LA) and LV] remain unclear. It should be noted that to compensate for chronic volume overload preoperatively, the left-sided heart chambers undergo reverse remodeling, which in turn contributes to the improvement in their dynamics postoperatively. Cardiac magnetic resonance imaging (CMRI) is a superior technique over other several testing methods for the measurement of cardiac chamber volumes (6).

We hypothesized that MVR improves the dynamics of left-sided cardiac chambers. In this study, we aimed to investigate the volumes of the LA and the LV before and after surgery; volumetric analysis was performed using CMRI after MVR.

\section{Patients and Methods}

From January 1, 2012 to December 31, 2018, 103 patients underwent mitral valve surgery for severe MR at our Institute. This retrospective study was limited to patients undergoing MVR with preoperative echocardiographic data of normal LV ejection fraction (LVEF) $>60 \%$. Those with concomitant tricuspid annuloplasty, patent foramen ovale closure, or pulmonary vein isolation for paroxysmal atrial fibrillation were eligible for inclusion. The exclusion criteria were concomitant coronary artery bypass grafting, a history of coronary artery disease, previous mitral valve intervention, mitral stenosis, aortic valve disease, infective endocarditis, congenital heart disease, cardiomyopathy, chronic atrial fibrillation, end-stage renal disease on hemodialysis, incomplete pre- or postoperative CMRI data, or refusal of patient consent. A total of 21 patients met the enrollment criteria and were analyzed in this study. The Ethics Committee of Nara Medical University approved this study (approval no.: 2307). 
CMRI acquisition. In this study, we accurately measured the LA and LV volumes and LV mass in patients with chronic MR by CMRI before and after mitral valve surgery, and we assessed both the structural and functional changes in the left-sided cardiac chambers after MVR. CMRI was performed around 1 month preoperatively and approximately 12 months postoperatively.

Patients were at rest and examined in the supine position using a 1.5-T scanner (Avanto; Siemens, Erlangen, Germany) with an 8element phased-array body coil. Cine images were acquired at a rate of 25 phases per cardiac cycle using segmented electrocardiogramtriggered, steady-state, free-precession cine imaging with echo sharing (true fast imaging with steady-state, free-precession sequence) from the same 8-10 contiguous short-axis planes with an 8 -mm section thickness and an inter-slice gap of $2 \mathrm{~mm}$, thereby covering the entire LV from the base to the apex, as described previously (7).

In all the patients, the following static LA volumes were measured:

- Maximum LA volume (LAVmax)

- LA volume immediately before atrial contraction (LAVpreA)

- Minimum LA volume (LAVmin)

Based on the aforementioned volumes, the following dynamic LA volumes were calculated:

- LA reservoir volume: LAVmax - LAVmin

- LA passive emptying volume: LAVmax - LAVpreA

- LA conduit volume: LV total stroke volume - LA reservoir volume

- LA booster pump volume: LAVpreA - LAVmin

The three LA phasic functions were expressed as the percentage contribution of respective phasic functions to the LV filling volume as follows:

- Reservoir function: LA passing emptying volume/LV total stroke volume

- Conduit function: LA conduit volume/LV total stroke volume

- Booster pump function: LA booster pump volume/LV total stroke volume

Surgical procedure. MVR was performed through full sternotomy. The approach to the mitral valve was through the atriotomy that was made on the right side of the LA. Valve repair was performed with triangular leaflet resection or chordal replacement using Gore-Tex sutures (Gore-Tex; W.L. Gore \& Associates, Inc., Flagstaff, AZ, USA) for posterior prolapsing or flailing leaflet. Anterior leaflet prolapse was commonly corrected using chordal replacement with Gore-Tex sutures. Mitral annuloplasty was usually accompanied by an annuloplasty ring (Cosgrove-Edwards Annuloplasty System, Carpentier-Edwards Physio II Annuloplasty Ring; Edwards Lifescience Corporation, Irvine, CA, USA). Moreover, myocardial protection was achieved with antegrade and retrograde cardioplegia. Transesophageal echocardiography was used routinely to assess the repaired valve intraoperatively. We found that residual MR was less than mild in all the patients.

Statistical analysis. Continuous variables are expressed as the mean and standard deviation or as the median and interquartile range. Categorical variables are presented as numbers and percentages. Measurements were compared using the Wilcoxon signed-rank test. A $p$-value of less than 0.05 was considered statistically significant. Statistical analyses were performed using SPSS for Windows, version 21.0 (Chicago, IL, USA).
Table I. Patient characteristics in hypopharyngeal cancer.

\begin{tabular}{|c|c|}
\hline Characteristic & Value \\
\hline Age, years $($ Mean $\pm S D)$ & $60.5 \pm 10.0$ \\
\hline Men, n (\%) & $10(47.6)$ \\
\hline $\mathrm{BSA}, \mathrm{m}^{2}(\mathrm{Mean} \pm \mathrm{SD})$ & $1.7 \pm 0.2$ \\
\hline \multicolumn{2}{|l|}{ NYHA class, $\mathrm{n}(\%)$} \\
\hline I & $3(14.3)$ \\
\hline II & $15(71.4)$ \\
\hline III & $2(9.5)$ \\
\hline IV & $1(4.8)$ \\
\hline Preoperative EF, \% & $68.8 \pm 5.0$ \\
\hline Preoperative LVDs, mm $($ Mean \pm SD $)$ & $35.9 \pm 5.9$ \\
\hline Operation time, $\min (\mathrm{Mean} \pm \mathrm{SD})$ & $384.5 \pm 100.1$ \\
\hline $\mathrm{BPT}, \min (\mathrm{Mean} \pm \mathrm{SD})$ & $200.6 \pm 48.0$ \\
\hline $\mathrm{CCT}, \min (\mathrm{Mean} \pm \mathrm{SD})$ & $143.8 \pm 37.2$ \\
\hline EuroSCORE II, \% (Mean \pm SD) & $1.8 \pm 0.5$ \\
\hline HT, n (\%) & $7(33.3)$ \\
\hline $\mathrm{DM}, \mathrm{n}(\%)$ & $0(0)$ \\
\hline $\mathrm{DL}, \mathrm{n}(\%)$ & $10(47.6)$ \\
\hline $\mathrm{CKD}, \mathrm{n}(\%)$ & $4(19.0)$ \\
\hline
\end{tabular}

BSA: Body surface area; NYHA: New York Heart Association; BPT: bypass time; CCT: cross-clamp time; HT: hypertension; DM: diabetes mellitus; DL: dyslipidemia; CKD: chronic kidney disease.

\section{Results}

Clinical characteristics. Patient characteristics are presented in Table I. The mean patient age was 60.5 years. Although all of the 21 patients had LVEF $>60 \%$ on transthoracic echocardiography preoperatively, five patients had LV endsystolic dimension $>40 \mathrm{~mm}$. No patients had persistent or permanent atrial fibrillation. However, one patient, who had had a history of paroxysmal atrial fibrillation, underwent concomitant pulmonary vein isolation. During follow-up after the surgery, all 21 patients have been in sinus rhythm.

Surgical data. Surgical data are shown in Table II. Seventeen patients had posterior mitral valve leaflet prolapse or flailing, which involved one or two scallops. Two patients had anterior leaflet prolapse, one had bileaflet prolapse, and one patient had a posterior commissure lesion. At our Institution, we have mainly employed chordal replacement with GoreTex sutures for anterior or posterior leaflet since 2015 . Tricuspid annuloplasty, closure of patent foramen ovale, and pulmonary vein isolation were concomitantly performed, as appropriate. No mortality or hospital re-admission for heart failure was reported. Postoperative residual regurgitation on transthoracic echocardiography was less than mild in all patients based on their latest clinical visit.

Structural and functional changes in the LV. In the 21 patients, LV end-diastolic volume index (LVEDVI), LV end- 
Table II. Surgical data.

\begin{tabular}{|c|c|c|c|c|c|}
\hline Patient & $\begin{array}{c}\text { Prolapse } \\
\text { lesions }\end{array}$ & $\begin{array}{l}\text { Surgical } \\
\text { procedure }\end{array}$ & Ring size $(\mathrm{mm})$ & $\begin{array}{l}\text { Residual } \\
\text { regurgitation }\end{array}$ & $\begin{array}{c}\text { Concomitant } \\
\text { procedures }\end{array}$ \\
\hline 1 & $\mathrm{P} 2$ & Cut and suture & Cosgrove ring (\#28) & None & None \\
\hline 2 & P3 & Cut and suture & Cosgrove ring (\#30) & Trivial & TAP \\
\hline 3 & P2 & Cut and suture & Cosgrove ring (\#30) & Trivial & TAP \\
\hline 4 & P3 & Cut and suture & Cosgrove ring (\#30) & Trivial & None \\
\hline 5 & P2-3 & Cut and suture & Cosgrove ring (\#32) & Trivial & None \\
\hline 6 & $\mathrm{P} 2-3$ & Cut and suture & Cosgrove ring (\#28) & Trivial & TAP PVI \\
\hline 7 & A1-2 & Cut and suture & None & Trivial & TAP \\
\hline 8 & P2 & Cut and suture & Cosgrove ring (\#30) & None & None \\
\hline 9 & P2 & Cut and suture +chordal replacement & Cosgrove ring (\#30) & Trivial & None \\
\hline 10 & P2 & Cut and suture & Cosgrove ring (\#34) & Trivial & None \\
\hline 11 & $\mathrm{P} 2$ & Chordal replacement & Cosgrove ring (\#32) & Trivial & None \\
\hline 12 & $\mathrm{P} 2$ & Chordal replacement & Cosgrove ring (\#36) & Trivial & None \\
\hline 13 & P2 & Chordal replacement & Cosgrove ring (\#30) & Trivial & None \\
\hline 14 & $\mathrm{~A} 2 \mathrm{P} 3$ & Chordal replacement & Cosgrove ring (\#30) & Trivial & TAP \\
\hline 15 & P2 & Chordal replacement & Cosgrove ring (\#32) & Trivial & TAP \\
\hline 16 & $\mathrm{~A} 2$ & Chordal replacement & Physio II ring (\#34) & Trivial & TAP \\
\hline 17 & $\mathrm{P} 2-3$ & Chordal replacement & Cosgrove ring (\#32) & Trivial & TAP \\
\hline 18 & $\mathrm{PC}$ & Suture & Physio II ring (\#32) & Trivial & None \\
\hline 19 & $\mathrm{P} 2$ & Chordal replacement & Cosgrove ring (\#32) & Trivial & PFOc \\
\hline 20 & $\mathrm{P} 2$ & Chordal replacement & Cosgrove ring (\#32) & Trivial & None \\
\hline 21 & P3 & Chordal replacement & Cosgrove ring (\#30) & Trivial & None \\
\hline
\end{tabular}

TAP: Tricuspid annuloplasty; PFOc: closure of patent foramen ovale; PVI: pulmonary vein isolation; Cosgrove ring: Cosgrove-Edwards Annuloplasty System; Physio II ring: Carpentier-Edwards Physio II Annuloplasty Ring.

systolic volume index (LVESVI), LVEF, and LV mass index (LVMI) were measured by CMRI. Preoperative LVEDVI and LVESVI were significantly reduced postoperatively, by $40 \%$ or more $(p<0.01)$ (Figure 1A and B). Preoperative LVEF decreased slightly postoperatively $(p=0.076)$ (Figure 1C); this finding reflected a less pronounced reduction in ESV than in EDV following surgery. LVMI was significantly lower postoperatively $(p<0.01)$ (Figure 1D).

Structural and functional changes in the LA. LAVmax, LAVpreA, and LAVmin were measured by CMRI, and LA reservoir volume, passive emptying volume, conduit volume, and booster pump volume were also calculated. Subsequently, LA reservoir function, conduit function, and booster pump function were assessed. The preoperative indexed LAVmax, LAVpreA and LAVmin significantly decreased postoperatively by $40 \%$ or more $(p=0.001)$ (Figure 2). Regarding the three LA phasic functions, no difference between preoperative and postoperative reservoir function was found $(p=0.103)$. The postoperative conduit function contributed more to the LV filling volume than did the preoperative conduit function, with a increase of more than $50 \%(p=0.007)$. Furthermore, the postoperative booster pump function contributed less to LV filling than did the preoperative booster pump function being lower by over a third $(p=0.013)$ (Figure 3).
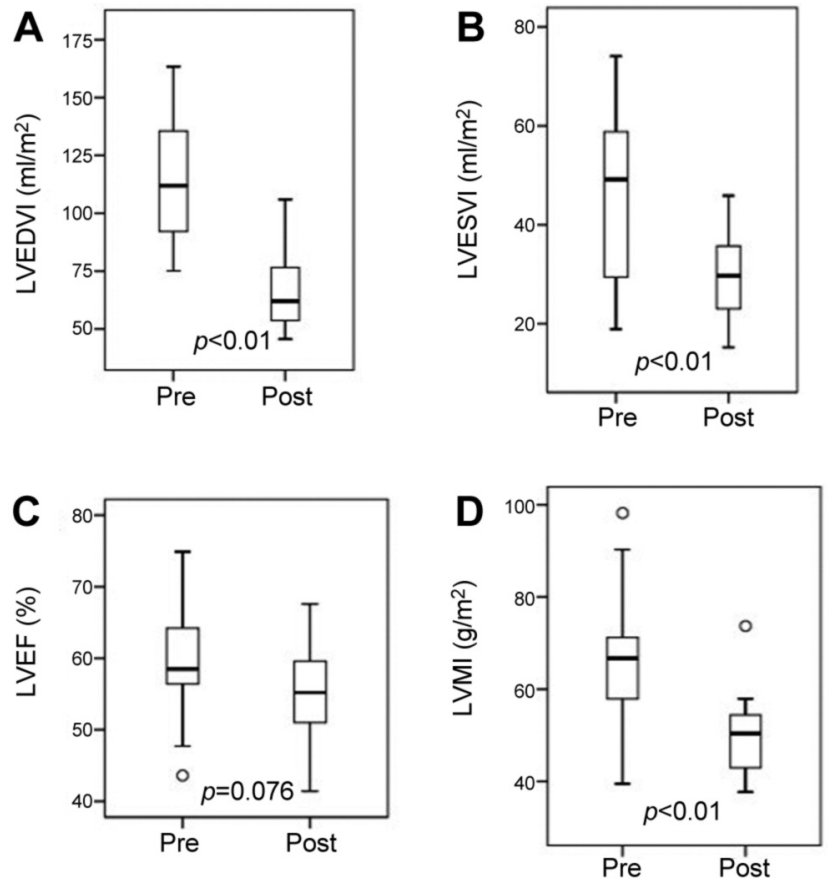

Figure 1. Boxplots showing the minimum, first quartile, median, third quartile, and maximum left ventricular end-diastolic volume index (LVEDVI) (A), left ventricular end-systolic volume index (LVESVI) (B), left ventricular ejection fraction $(L V E F)(C)$, and left ventricular mass index (LVMI) (D) before (Pre) and after (Post) mitral valve repair. 

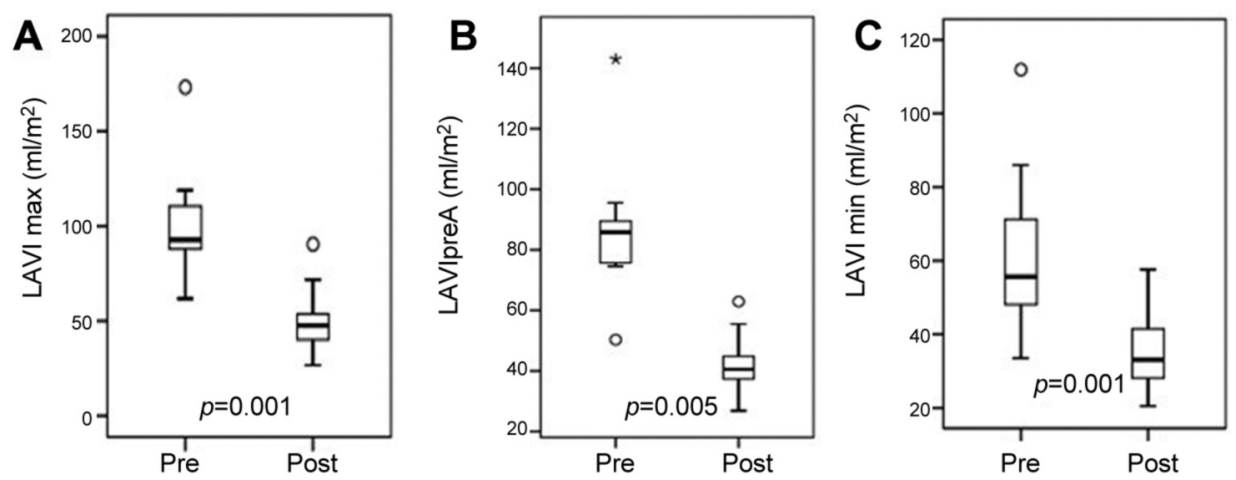

Figure 2. Boxplots showing the minimum, first quartile, median, third quartile, and maximum static volumes of the left atrium (LA) before (Pre) and after (Post) mitral valve repair. A: Maximum LA volume (LAVmax). B: LA volume immediately before atrial contraction (LAVpreA). C: minimum $L A$ volume (LAVmin). All volumes were indexed to body surface area.
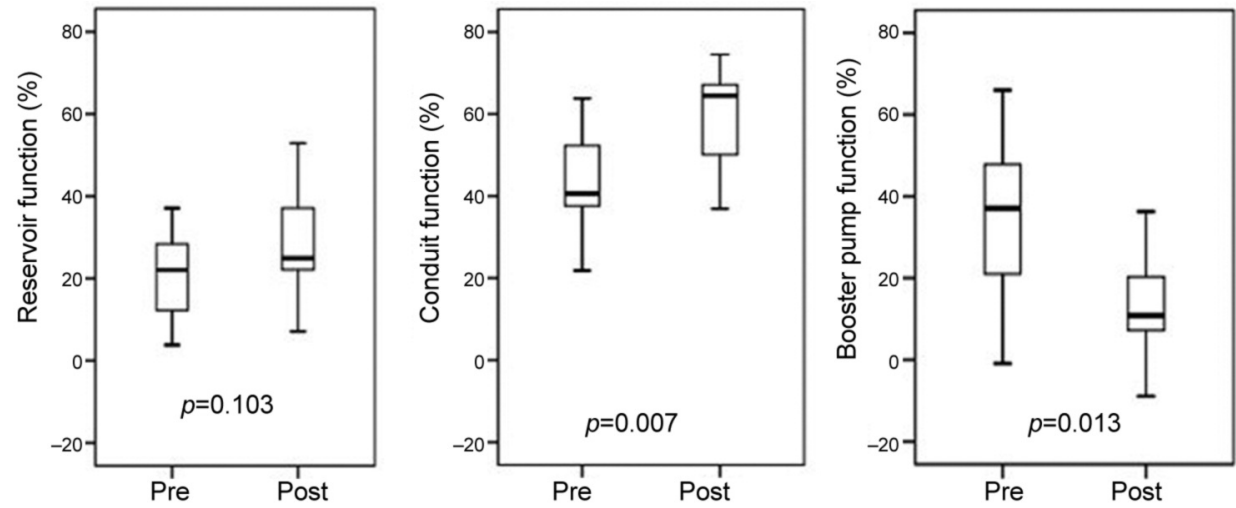

Figure 3. Contribution of each phasic function of the left atrium (LA) to the total stroke volume of the left ventricle. No difference in the reservoir function before (Pre) and after (Post) mitral valve repair was found. Postoperative conduit function had a significantly greater contribution, whereas postoperative booster pump function had significantly less contribution to left ventricular filling than preoperatively.

\section{Discussion}

Studies have reported on the excellent long-term outcomes of MVR $(1,2)$; thus, it has become the gold standard surgery for chronic primary MR. However, little is known regarding the structural and functional changes in the left-sided cardiac chambers after surgery. Some researchers have shown postoperative decrease in LVEF despite LV reverse remodeling after the surgery $(8,9)$. It should be confirmed that the seeming structural improvement in the LV is linked to true improvement in cardiac performance.

Patients with indications for MVR are usually in a stable compensated condition for chronic volume overload. The compensatory mechanism results in dilatation of the leftsided cardiac chambers. Thus, in our study, we measured the LA and LV volumes by CMRI, which can accurately measure cardiac chamber volumes (6). Moreover, the LA function has three phases, acting as a reservoir in systole, as a conduit in early diastole, and as a booster pump in late diastole (10). These LA phasic functions can be assessed by volumetric evaluation. Considering that left-sided heart failure syndrome is associated with the failure of the LA to compensate for LV dysfunction, it is essential to investigate the changes in LA functions in the three phases after MVR.

LV following MVR. Preoperative LV chamber remodeling with eccentric hypertrophy was reversed because of a significant decrease in LV preload with reduction in EDV, ESV, and LV mass in almost all patients after MVR. However, despite the substantial LV reverse remodeling, LVEF decreased from $58.5 \%$ at baseline to $55.2 \%$ at followup. As other studies have shown, the postoperative reduction in LVEF is because afterload-dependent LVESV decreases less pronouncedly than preload-dependent $\operatorname{LVEDV}(11,12)$, 
which was also demonstrated in our study patients. This finding may indicate that the preoperative relationship between increased LV preload, reduced LV afterload, and compromised LV contractility was corrected by surgery, resulting in the normalization of preload and afterload. Nevertheless, predicting the postoperative LV function in a patient before MVR remains challenging because of the difficulty in accurately assessing the LV function in a volume-overload condition. Therefore, postoperative LV dysfunction may still occur in some patients who are indicated for MVR according to the current guidelines.

Witkowski et al. (13) and Kim et al. (14) demonstrated that preoperative LV global longitudinal strain, which is assessed by speckle-tracking echocardiographic analysis, is an independent predictor of long-term outcomes after MVR. The LV myocardium consists of circumferential fibers in the mid-wall layer and longitudinal fibers in the subendocardial and subepicardial layers $(15,16)$. It has been suggested that the subendocardial fibers may play a central role in the descent motion of the mitral annulus to the apex during systole and that longitudinal myocardial function may be impaired earlier than circular myocardial function in cardiac disorders because of the subendocardial localization of the longitudinal fibers. Therefore, preoperative LV global longitudinal strain may be a promising predictor of long-term outcomes following MVR. Moreover, treatment of severe MR with percutaneous devices has recently been developed as an alternative to surgery. Ipek et al. showed changes in LV longitudinal strain from baseline to 12-month follow-up in two groups: MitraClip and MVR groups. A significant postoperative reduction in LV longitudinal strain in the MVR group was observed, whereas no change was noted in the MitraClip group (17). Some factors in surgical repair possibly contributed to such postoperative LV dysfunction.

LA following MVR. Preoperative LA adverse remodeling was also reversed by a significant reduction in LA preload with decreased static LA volumes, including LAVmax, LAVpreA, and LAVmin. In our study, each phasic function in the three phases was investigated and the dynamic LA volumes were assessed because the LA function contributes significantly to the onset of left heart failure syndrome. The postoperative contribution of reservoir function to the LV stroke volume was $25 \%$, which was not significantly different from the preoperative contribution. While postoperative conduit function increased to $65 \%$, booster pump function was reduced to $11 \%$. Prioli et al., who investigated the three phasic functions in the LA in healthy individuals by echocardiography, showed that reservoir function contributes $38 \%$, conduit function $36 \%$, and booster pump function $26 \%$ to the LV stroke volume (18). In our patients, the postoperative findings of the three phasic functions corresponded to those of the restrictive rather than the healthy individuals in Prioli et al.'s study. Thus, postoperative structural improvement in the LA might not necessarily reflect functional improvement.

Borg et al. studied LA mechanisms in patients with MR and in healthy controls using echocardiography; they showed that all static LA volumes and dynamic LA volumes in the three LA phases significantly increased in the MR group and that the conduit function produced approximately $70 \%$ of the total LV stroke volume (19). Ren et al. also demonstrated an augmentation of the LA conduit function in severe MR (20). In our patients, preoperative conduit function provided $41 \%$ of the total stroke volume. In a normal heart, an increase in atrial preload, e.g. during exercise, results in an augmentation of LA reservoir and booster pump functions, whereas no augmentation of conduit function is noted (21). Thus, in patients with chronic severe MR, including those who are asymptomatic, the LA phasic functions might be already deranged because of volume overload. In addition to such derangement of LA function, the LA may be burdened by LV dysfunction due to LV volume overload, an annuloplasty ring may impede mitral flow in diastole, and left atriotomy and manipulation of the LA may affect LA performance following MVR. However, determining how the LA with preoperative compensation for chronic volume overload adapts after surgery, resulting in restoration of the normal volumeloading condition, remains challenging. Nevertheless, Ring et al. proposed that a quantitative assessment of LA function, particularly the total LA emptying fraction, may guide the optimum timing of surgery for chronic MR (22). The LA mechanisms may play an important role in cardiac performance after surgery for chronic MR; hence, attention to the LA may be crucial in decision-making regarding the optimal timing of MVR.

Limitations. This was a single-center retrospective study and thus had well-known inherent limitations. The number of patients was small, which can be attributed to the strict study criteria. The patients had to be in sinus rhythm and had to undergo CMRI for the accurate measurement of the leftsided heart chamber volumes both pre- and postoperatively. Moreover, the main purpose of this study was to evaluate the structural and functional changes in the LV and LA after MVR in patients with chronic severe MR, and long-term outcome measures were beyond the scope of our investigation. Nevertheless, larger studies are needed to confirm our results, and the prognostic implications of LV and LA reverse remodeling after surgery should be demonstrated with a longer clinical follow-up.

\section{Conclusion}

Functional restoration of the LA and LV after a successful MVR may not be necessarily associated with their structural 
improvement, particularly in regard to the LA. However, the findings in our study warrant further investigations.

\section{Conflicts of Interest}

All Authors declare no conflicts of interest associated with this study.

\section{Authors' Contributions}

Conception and design: Yoshihiro Hayata, Shigeki Taniguchi. Administrative support: Yoshihiro Hayata, Takehisa Abe, Tomoaki Hirose, Keigo Yamashita, Shun Hiraga, Ryohei Fukuba, Junnichi Takemura, Tomoya Nakano. Provision of study materials or patients: Yoshihiro Hayata, Shigeki Taniguchi. Collection and assembly of data: Yoshihiro Hayata, Tomoya Nakano. Data analysis and interpretation: Yoshihiro Hayata, Shigeki Taniguchi, Noriyoshi Sawabata. Article writing and final article approval: All Authors.

\section{Acknowledgements}

The Authors would like to thank Editage (www.editage.com) for English language editing.

\section{References}

1 Chikwe J, Toyoda N, Anyanwu AC, Itagaki S, Egorova NN, Boateng P, El-Eshmawi A and Adams DH: Relation of mitral valve surgery volume to repair rare, durability, and survival. J Am Coll Cardiol 69(19): 2397-2406, 2017. PMID: 28476349. DOI: $10.1016 /$ j.jacc.2017.02.026

2 David TE, David CM, Tsang W, Lafreniere-Roula M and Manlhiot C: Long-term results of mitral valve repair for regurgitation due to leaflet prolapse. J Am Coll Cardiol 74(8): 1044-1053, 2019. PMID: 31439213. DOI: 10.1016/j.jacc. 2019.06.052

3 Nishimura RA, Otto CM, Bonow RO, Carabello BA, Ewin JP $3^{\text {rd }}$, Fleisher LA, Jneid H, Mack MJ, McLeod CJ, O'Gara PT, Rigolin VH, Sundt TM, 3rd and Thompson A: 2017 AHA/ACC focused update of the 2014 AHA/ACC guideline for the management of patients with valvular heart disease: A report of the American College of Cardiology/American Heart Association Task Force on clinical practice guidelines. Circulation 135(25): e1159-e1195, 2017. PMID: 28298458. DOI: 10.1161/CIR. 0000000000000503

4 Baumgartner H, Falk V, Bax JJ, De Bonis M, Hamm C, Holm PJ, Iung B, Lancellotti P, Lansac E, Muñoz DR, Rosenhek R, Sjögren J, Mas PT, Vahanian A, Walther T, Wendler O, Windecker S, Zamorano JL and ESC Scientific Document Group: 2017 ESC/EACTS guidelines for the management of valvular heart disease: the task force for the management of valvular heart disease of the European Society of Cardiology (ESC) and the European Association for Cardio-thoracic Surgery (EACTS). Eur Heart J 38(36): 2739-2791, 2017. DOI: 10.1093/ eurheartj/ehx391

5 Bonow RO: Left atrial function in mitral regurgitation. Guilt by association. JACC Cardiovasc Imaging 7(3): 233-235, 2014. PMID: 24651098. DOI: 10.1016/j.jcmg.2014.01.009
6 Grothues F, Smith GC, Moon JCC, Bellenger NG, Collins P, Klein $\mathrm{HU}$ and Pennel DJ: Comparison of interstudy reproducibility of cardiovascular magnetic resonance with two-dimensional echocardiography in normal subjects and in patients with heart failure or left ventricular hypertrophy. Am J Cardiol 90(1): 29-34, 2002. PMID: 12088775. DOI: 10.1016/s0002-9149(02)02381-0

7 Okayama S, Uemura S, Soeda T, Onoue K, Somekawa S, Ishigami K, Watanabe M, Nakajima T, Fujimoto S and Saito Y: Clinical significance of papillary muscle late enhancement detected via cardiac magnetic resonance imaging in patients with single old myocardial infarction. Int J Cardiol 146(1): 73-79, 2011. PMID: 20471120. DOI: 10.1016/j.ijcard.2010.04.037

8 Pandis D, Grapsa J, Athanasiou T, Punjabi $\mathrm{P}$ and Nihoyannopoulos P: Left ventricular remodeling and mitral valve surgery: Prospective study with real-time 3-dimensional echocardiography and speckle tracking. J Thorac Cardiovasc Surg 142(3): 641-649, 2011. PMID: 21277588. DOI: 10.1016/ j.jtcvs.2010.11.030

9 Quintana E, Suri RM, Thalji NM, Daly RC, Dearani JA, Burkhart HM, Li Z, Enriquez-Sarano M and Schaff HV: Left ventricular dysfunction after mitral valve repair-the fallacy of "normal" preoperative myocardial function. J Thorac Cardiovasc Surg 148(6): 2752-2762, 2014. PMID: 25173130. DOI: 10.1016/ j.jtcvs.2014.07.029

10 Payne RM, Stone HL and Engelken EJ: Atrial function during volume loading. J Appl Physiol 31(3): 326-331, 1971. PMID: 5111850. DOI: 10.1152 jappl.1971.31.3.326

11. Gaasch WH and Meyer TE: Left ventricular response to mitral regurgitation: implications for management. Circulation 118(22): 2298-2303, 2008. PMID: 19029478. DOI: 10.1161/ CIRCULATIONAHA.107.755942

12 Witkowski TG, Thomas JD, Delgado V, van Rijnsoever E, Ng AC, Hoke U, Ewe SH, Auger D, Yiu KH, Holman ER, Klautz RJM, Schalij MJ, Bax JJ and Marsan NA: Changes in left ventricular function after mitral valve repair for severe organic mitral regurgitation. Ann Thorac Surg 93(3): 754-760, 2012. PMID: 22296981. DOI: 10.1016/j.athoracsur.2011.11.034

13 Witkowski TG, Thomas JD, Debonnaire PJ, Delgado V, Hoke U, Ewe SH, Versteegh MIM, Holman ER, Schalij MJ, Bax JJ, Klautz RJM and Marsan NA: Global longitudinal strain predicts left ventricular dysfunction after mitral valve repair. Eur Heart J Cardiovasc Imaging 14(1): 69-76, 2013. PMID: 22848021. DOI: $10.1093 /$ ehjci/jes155

14 Kim HM, Cho GY, Hwang IC, Choi HM, Park JB, Yoon YE, et al. Myocardial strain in prediction of outcomes after surgery for severe mitral regurgitation. JACC Cardiovasc Imaging 11(9): 1235-1244, 2018. PMID: 29778855. DOI: 10.1016/j.jcmg. 2018.03.016

15 Sengupta PP, Krishnamoorthy VK, Korinek J, Narula J, Vannan MA, Lester SJ, Tajik JA, Seward JB, Khandheria BK and Belohlavek M: Left ventricular form and function revisited: applied translational science to cardiovascular ultrasound imaging. J Am Soc Echocardiogr 20(5): 539-551, 2007. PMID: 17485001. DOI: 10.1016/j.echo.2006.10.013

16 Leitman M, Lysiansky M, Lysyansky P, Friedman Z, Tyomkin V, Fuchs T, Adam D, Krakover R and Vered Z: Circumferential and longitudinal strain in 3 myocardial layers in normal subjects and in patients with regional left ventricular dysfunction. J Am Soc Echocardiogr 23(1): 64-70, 2010. PMID: 20122496. DOI: 10.1016/j.echo.2009.10.004 
17 Ipek EG, Singh S, Viloria E, Feldman T, Grayburn P, Foster E and Qasim A: Impact of the MitraClip procedure on left atrial strain and strain rate. Circ Cardiovasc Imaging 11(3): e006553, 2018. PMID: 29535132. DOI: 10.1161/CIRCIMAGING. 117.006553

18 Prioli A, Marino P, Lanzoni L and Zardini P: Increasing degrees of left ventricular filling impairment modulate left atrial function in humans. Am J Cardiol 82(6): 756-761, 1998. PMID: 9761086. DOI: 10.1016/s0002-9149(98)00452-4

19 Borg AN, Pearce KA, Williams SG and Ray SG: Left atrial function and deformation in chronic primary mitral regurgitation. Eur J Echocardiogr 10(7): 833-840, 2009. PMID: 19531539. DOI: $10.1093 /$ ejechocard/jep085

20 Ren B, de Groot-de Laat LE and Geleijnse ML: Left atrial function in patients with mitral valve regurgitation. Am J Physiol Heart Circ Physiol 307(10): H1430-H1437, 2014. PMID: 25217651. DOI: 10.1152/ajpheart.00389.2014
21 Toutouzas K, Trikas A, Pitsavos C, Barbetseas J, Androulakis A, Stefanadis $\mathrm{C}$ and Toutouzas P: Echocardiographic features of left atrium in elite male athletes. Am J Cardiol 78(11): 1314-1317, 1996. PMID: 8960602. DOI: 10.1016/s0002-9149(96)00622-4

22 Ring L, Rana BS, Wells FC, Kydd AC and Dutka DP: Atrial function as a guide to timing of intervention in mitral valve prolapse with mitral regurgitation. JACC Cardiovasc Imaging 7(3): 225-232, 2014. PMID: 24529886. DOI: 10.1016/j.jcmg. 2013.12.009

Received June 4, 2020

Revised July 2, 2020

Accepted July 6, 2020 